\title{
PPP2R2B hypermethylation causes acquired apoptosis deficiency in systemic autoimmune diseases
}

\author{
Iris K. Madera-Salcedo, ${ }^{1}$ Beatriz E. Sánchez-Hernández, ${ }^{2}$ Yevgeniya Svyryd, ${ }^{2}$ \\ Marcela Esquivel-Velázquez, ${ }^{1}$ Noé Rodríguez-Rodríguez, ${ }^{1}$ María Isabel Trejo-Zambrano, ${ }^{1}$ \\ H. Benjamín García-González, ' Gabriela Hernández-Molina, ${ }^{1}$ Osvaldo M. Mutchinick, ${ }^{2}$ \\ Jorge Alcocer-Varela, ${ }^{1}$ Florencia Rosetti, ${ }^{1}$ and José C. Crispín'
}

'Department of Immunology and Rheumatology and 2Department of Genetics, Instituto Nacional de Ciencias Médicas y Nutrición Salvador Zubirán, Mexico City, Mexico.

\begin{abstract}
Chronic inflammation causes target organ damage in patients with systemic autoimmune diseases. The factors that allow this protracted response are poorly understood. We analyzed the transcriptional regulation of $P P P 2 R 2 B$ (B55 $\beta$ ), a molecule necessary for the termination of the immune response, in patients with autoimmune diseases. Altered expression of B55 $\beta$ conditioned resistance to cytokine withdrawal-induced death (CWID) in patients with autoimmune diseases. The impaired upregulation of B55 $\beta$ was caused by inflammation-driven hypermethylation of specific cytosines located within a regulatory element of PPP2R2B preventing CCCTC-binding factor binding. This phenotype could be induced in healthy T cells by exposure to TNF- $\alpha$. Our results reveal a gene whose expression is affected by an acquired defect, through an epigenetic mechanism, in the setting of systemic autoimmunity. Because failure to remove activated T cells through CWID could contribute to autoimmune pathology, this mechanism illustrates a vicious cycle through which autoimmune inflammation contributes to its own perpetuation.
\end{abstract}

Conflict of interest: The authors have declared that no conflict of interest exists.

Copyright: (c) 2019 American Society for Clinical Investigation

Submitted: November 27, 2018

Accepted: July 18, 2019

Published: August 22, 2019

Reference information: /Cl Insight. 2019;4(16):e126457. https://doi. org/10.1172/jici.nsight.126457.

\section{Introduction}

A common hallmark of systemic autoimmune diseases is the loss of immune tolerance and the development of a chronic autoimmune response revealed by the presence of autoantibodies and increased numbers of activated $\mathrm{T}$ cells (1). The factors that underlie chronic immune activation are poorly understood, but constant and ubiquitous presence of antigens and regulatory $\mathrm{T}$ cell dysfunction have been proposed to contribute (2). Patients in which regulatory mechanisms are in place tend to have milder disease. For example, patients with systemic lupus erythematosus (SLE) whose T cells exhibit a gene expression profile indicative of exhaustion have a better prognosis than patients with no transcriptional signs of T cell exhaustion $(3,4)$. Therefore, a better understanding of the mechanisms that limit the magnitude and length of immune responses and the identification of those that fail during autoimmunity is warranted.

Protein phosphatase 2A (PP2A) is a ubiquitously expressed serine/threonine phosphatase involved in a large number of fundamental cellular processes, including apoptosis (5). PP2A is a heterotrimeric enzyme composed of a highly conserved catalytic subunit (PP2A C), a scaffold subunit (PP2A A), and a variety of regulatory subunits (PP2A B) that associate with the PP2A A/C heterodimer in a mutually exclusive manner. The regulatory subunits of PP2A are particularly relevant because they determine the specificity and subcellular location of the holoenzyme (6). PP2A B55 $\beta$ (henceforth referred to as B55 $\beta$ ) is induced in activated $\mathrm{T}$ cells by cytokine deprivation, and its presence is necessary and sufficient for $\mathrm{T}$ cells to undergo cytokine withdrawal-induced death (CWID) through poorly understood mechanisms (7). The expression of B55 $\beta$, tightly regulated at the transcriptional level, is deficient in patients with SLE whose T cells are resistant to apoptosis (7). Thus, failed induction of B55 $\beta$ could contribute to the perpetuation of $\mathrm{T}$ cell activation in patients with SLE.

The phenotype and behavior of $\mathrm{T}$ cells from patients with autoimmune diseases is grossly abnormal, and, in many cases, altered gene expression has been identified as a culprit (8). Genome-wide association studies (GWAS) have linked a number of loci with different autoimmune diseases (9). This has allowed the 
identification of genes whose abnormal expression might be facilitated by risk-conferring genetic variants (10). Because immune cells dramatically modify their transcriptional profile in response to environmental stimuli, it has been hard to distinguish the contribution of preexisting genetic variants from the effects imposed by an abnormal milieu. It is reasonable to assume that the behavior of immune cells represents the result of repetitive adaptations to the environment, biased in a qualitative and quantitative manner by the genetic elements variably present in each individual.

In this work, we have investigated the mechanisms that underlie defective B55 $\beta$ expression in patients with SLE and other autoimmune diseases. We have analyzed the genetic and epigenetic factors that regulate the transcription of $P P P 2 R 2 B$, the gene that encodes $B 55 \beta$. We provide data that demonstrate that, in patients with systemic autoimmune diseases, $P P P 2 R 2 B$ transcription is affected by an epigenetic mechanism driven by inflammation that hampers the binding of CCCTC-binding factor (CTCF) to its target region. Moreover, we show that defective expression of B55 $\beta$ can be induced in healthy $\mathrm{T}$ cells by the action of a proinflammatory environment. Our results illustrate how a pathological milieu can affect $\mathrm{T}$ cell behavior, generating a vicious cycle that perpetuates autoimmunity.

\section{Results}

The regulation of B55 $\beta$ expression is altered in patients with autoimmune diseases. B55 $\beta$ is induced by cytokine withdrawal in activated T cells from humans and mice (7). Its expression is necessary and sufficient for $\mathrm{T}$ cells to undergo CWID (7). To explore this mechanism in humans with autoimmune diseases, we isolated peripheral blood $\mathrm{T}$ cells from healthy donors (HDs) and patients with SLE, rheumatoid arthritis (RA), and primary Sjögren's syndrome (SS) (Supplemental Table 1; supplemental material available online with this article; https://doi.org/10.1172/jci.insight.126457DS1). T cells were activated in vitro with plate-bound anti-CD3 and anti-CD28 and expanded in the presence of IL-2 (11). Ten days after activation, cells were counted, washed, and resuspended in fresh RPMI devoid of IL-2 (Supplemental Figure 1). To examine the kinetics of B55 $\beta$ transcription, RNA was isolated from T cells before (basal) and after IL-2 deprivation, and quantitative PCR (qPCR) was performed. Expression of B55 $\beta$ in activated T cell blasts before IL-2 withdrawal was similar between HDs and patients with RA and SS. However, $P P P 2 R 2 B$ mRNA abundance was significantly lower in patients with SLE than in HDs $(0.50$-fold, $P=0.024$; Figure $1 \mathrm{~A})$. As expected, 2 days after IL-2 withdrawal, B55 $\beta$ levels had increased $\sim 3$-fold in HDs but remained low in patients with SLE (0.36-fold compared with HDs, $P=0.002)$. The kinetics of B55 $\beta$ expression were conserved in patients with SS, who in fact had higher levels than HDs 48 hours after IL-2 deprivation, whereas T cells from patients with RA exhibited a small increase in B55 $\beta$ that was not statistically different from the response of HDs (Figure 1A). When PPP $2 R 2 B$ mRNA levels were paired to compare the response of each individual sample to IL-2 withdrawal, HDs and patients with SS exhibited the expected behavior as B55 $\beta$ levels increased $2.27 \pm 0.35$-fold $(P=0.005)$ and $3.36 \pm 0.76$-fold $(P=0.007)$, respectively. However, the response of B55 $\beta$ to IL-2 withdrawal was heterogeneous in patients with SLE and RA; although the gene was induced in some samples, it showed no response in $\mathrm{T}$ cells from a large fraction of the patients (Figure $1, \mathrm{~B}$ and $\mathrm{C}$ ). As a result, the mean $P P P 2 R 2 B$ mRNA abundance was not statistically different before (basal) and after IL-2 withdrawal (SLE, $P=0.066$; RA, $P=0.064$ ) (Figure $1 \mathrm{~B}$ ). To assess the incidence of impaired $\mathrm{B} 55 \beta$ induction in response to IL-2 withdrawal, we divided the samples into 3 groups: (a) normal B55 $\beta$ induction, defined as an increment of $>1.5$-fold at 24 hours of IL-2 deprivation; (b) no change, defined as B55 $\beta$ levels between 1.5- and 0.5-fold at 24 hours of IL-2 deprivation; (c) decrease, defined as B55 $\beta$ levels $<0.5$-fold at 24 hours of IL-2 deprivation. As shown in Figure 1C, IL-2 deprivation induced an increase in B55 $\beta$ expression in $70 \%$ of HDs but only in $52 \%$ of patients with RA, $50 \%$ of patients with SLE, and $58 \%$ of patients with SS. Moreover, whereas B55 $\beta$ levels decreased only in 1 (out of 20) HD, they decreased in $19 \%, 25 \%$, and $16 \%$ of patients with RA, SLE, and SS, respectively. These differences were statistically significant (Figure 1C).

Defective B55 $\beta$ regulation is associated with impaired CWID. To determine the functional effects of the abnormal B55 $\beta$ expression kinetics, we analyzed apoptosis induced by cytokine withdrawal (CWID) in $\mathrm{T}$ cells from HDs and patients with autoimmune diseases (AID). Cells from patients with systemic AID exhibited a tendency to die less when subjected to CWID. This was particularly obvious when $\mathrm{CD} 4^{+}$and $\mathrm{CD}^{+} \mathrm{T}$ cells were analyzed separately (Figure 2A). To determine whether defective induction of B55 $\beta$, significantly more common in $\mathrm{T}$ cells from patients with AID, was involved in resistance to apoptosis, we divided the samples from the patients into 2 groups: (a) those with normal induction ( $>1.5$-fold) of B55 $\beta$ 
A

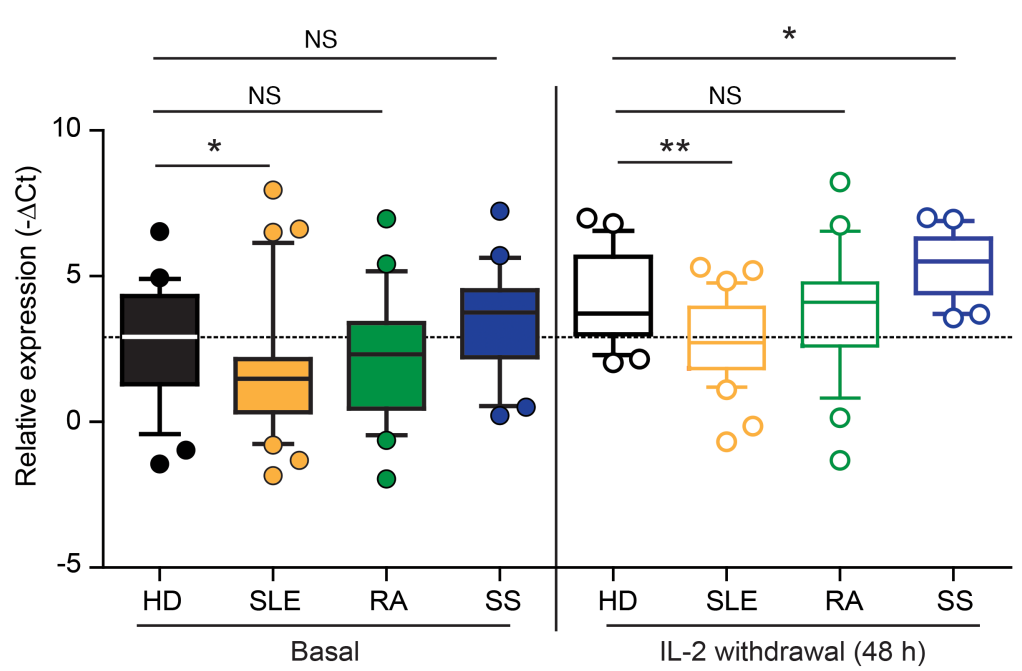

C

Control

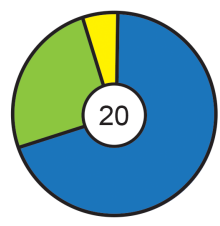

SLE

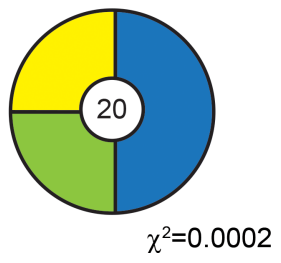

RA

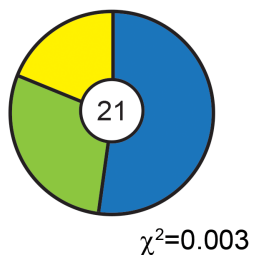

B

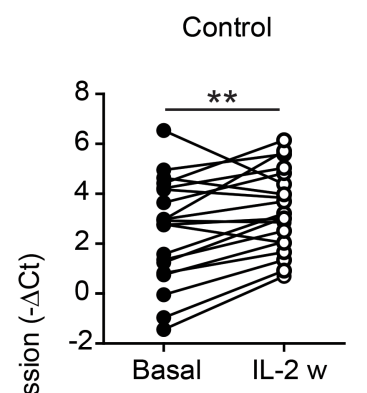

RA

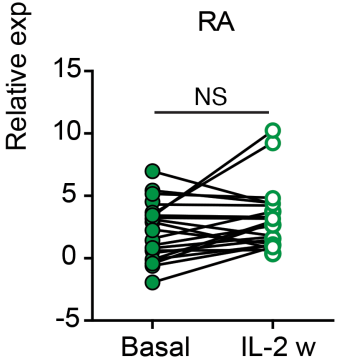

B55 $\beta$ induction

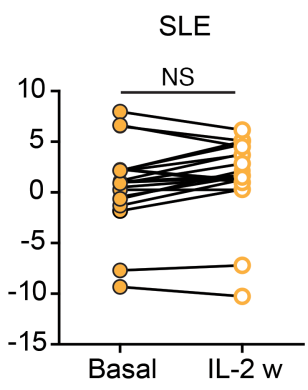

Figure 1. Expression and induction of B55 $\beta$ is impaired in T cells from patients with systemic autoimmune diseases. (A) Abundance of the B55 $\beta$ transcript was quantified in activated T cell blasts from healthy donors (HDs) and patients with systemic lupus erythematosus (SLE), rheumatoid arthritis (RA), and Sjögren's syndrome (SS), before (basal) and 48 hours after IL-2 withdrawal. Data are presented as median and quartiles (25th to 75th). Whiskers represent the 10th and 90th percentiles, and outliers are indicated by circles (HD basal vs. SLE basal $P=0.024 ;$ HD 48 hours vs. SLE 48 hours $P=0.002 ;$ HD 48 hours vs. SS 48 hours $P=0.012$; Mann-Whitney $U$ test) (HD $n=25$; SLE $n=32$; RA $n=28$; SS $n=21$ ). (B) Induction of B55 $\beta$ induced by IL-2 withdrawal (24 hours) is shown (HD basal vs. 24 hours $P=0.005$; SLE basal vs. 24 hours $P=0.066$; RA basal vs. 24 hours $P=0.064$; SS basal vs. 24 hours $P=0.007$, paired two-tailed $t$ test) (HD $n=20$; SLE $n=20$; RA $n=21$; SS $n=16$ ). (C) The proportion of controls and patients with increased B55 $\beta$ expression after IL-2 withdrawal is shown. The number in the pie chart indicates the number of controls or patients included in the analysis. Under the corresponding pie chart, $\chi^{2}$ of the distribution ratio (increase/no change/decrease) of HDs versus each autoimmune disease is indicated. IL-2 w, IL-2 withdrawal.

24 hours after IL-2 withdrawal and (b) those with defective induction. As shown in Figure 2B, T cells from patients that displayed a normal B55 $\beta$ upregulation underwent apoptosis with identical kinetics as cells from HDs. In sharp contrast, defective B55 $\beta$ induction was associated with marked resistance to CWID. In concordance, a positive correlation between B55 $\beta$ induction at 24 hours and $\mathrm{T}$ cell apoptosis at 48 hours was observed (Figure 2C; $r=0.39 ; P=0.004$ ).

Collectively, these results indicate that the correct regulation of B55 $\beta$ during cytokine withdrawal is essential for activated $\mathrm{T}$ cells to undergo apoptosis and that defective expression of this molecule is involved in $\mathrm{T}$ cell resistance to apoptosis in the setting of AID.

The length of the $C A G$ repeat element of PPP2R $2 B$ is normal in patients with $A I D$. In humans, $P P P 2 R 2 B$, the gene that encodes $\mathrm{B} 55 \mathrm{~B}$, is located in the long arm of chromosome 5 . There are no reports of associations between PPP2R2B genetic variants and AID. However, the gene contains a CAG trinucleotide in its $5^{\prime}$ untranslated region (UTR) that has been reported to affect the abundance of the B55 $\beta$ transcript (12). In fact, expansions of the CAG trinucleotide cause type 12 spinocerebellar ataxia, a rare neurodegenerative disease (13), probably by facilitating expression of B55 $\beta$ in neurons $(12,14)$. In concordance, shorter alleles with 5 to 7 CAG repeats have been associated with decreased B55 $\beta$ expression and linked to Alzheimer's disease in Taiwanese patients (15). Therefore, the available data suggest that the length of the CAG repeat element in the $5^{\prime}$ UTR of PPP2R2B modulates the cellular abundance of B55 $\beta$.

To determine whether the defective expression of B55 $\beta$ in T cells from patients with AID was related to the presence of short CAG repeat alleles, we quantified the number of CAG repeats in HDs and in patients 
A

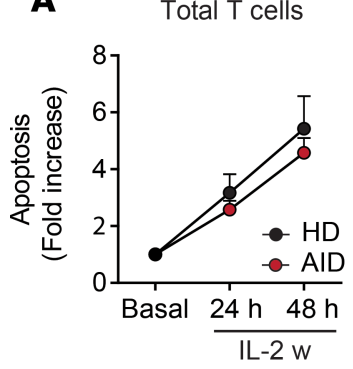

B

Total T cells

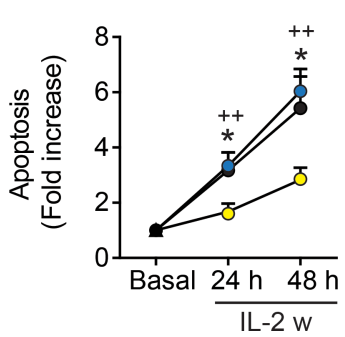

CD4 T cells

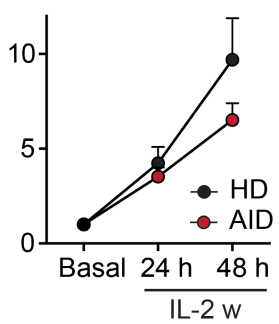

CD4 T cells

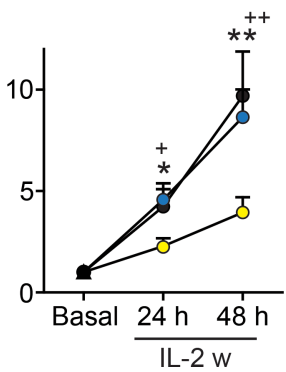

CD8 T cells

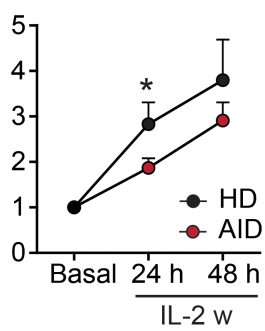

CD8 T cells

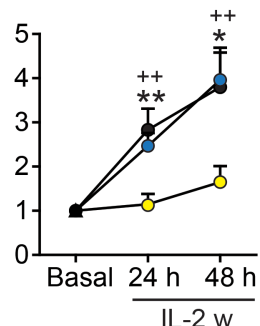

C

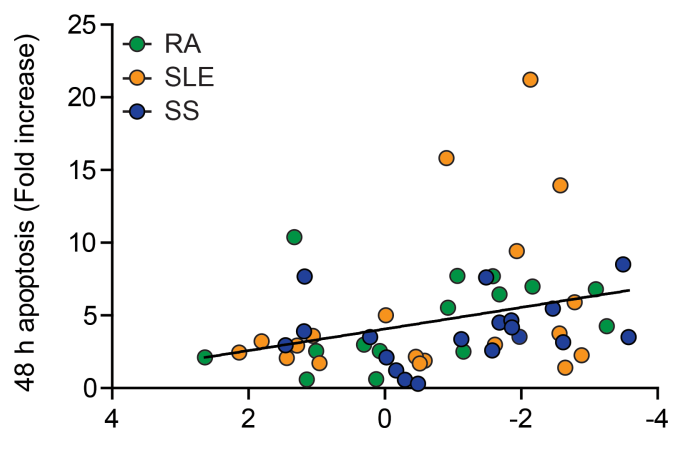

B55 $\beta$ induction $(\log 2)$

Figure 2. CWID is impaired in T cells that fail to upregulate B55ß. (A) Apoptosis was quantified by flow cytometry in activated T lymphoblasts from patients with AID and HDs, before (basal) and after IL-2 withdrawal (24 and 48 hours). Results are expressed as mean + SEM of annexin $\mathrm{V}^{+}$DAPI- cells. ${ }^{*} P$ $<0.05$, two-tailed $t$ test $(\mathrm{HD} n=19$; AID $n=59)$. (B) Apoptosis was compared in T cell subsets from HDs $(n=19)$ and from patients with AID with normal ( $n$ $=30)$ or defective $(n=25)$ B $55 \beta$ upregulation at 24 hours of IL $-2 \mathrm{w} .+P<0.05$ vs. HDs; $++P<0.01$ vs. HDs; ${ }^{* *} P<0.001$ vs. AID with normal B55 $\beta$ induction; 2-way ANOVA with Bonferroni's posttest. (C) Correlation between B55 $\beta$ induction at 24 hours (fold change over basal) and T cell apoptosis at 48 hours (fold change over basal). Spearman's $r=0.39$, and $P=0.004$.

with SLE and RA (Figure 3A). As shown in Figure 3B, the CAG repeat allele frequency was not different between HDs and patients with AID and was similar to the distribution reported in healthy populations (13). Of note, we did not detect any allele with fewer than 9 repeats (Supplemental Table 2). Moreover, when we compared the allele frequency between individuals with normal and defective B55 $\beta$ induction, no differences were observed (Figure 3C). These results indicate that the defects in B55 $\beta$ upregulation in response to IL-2 deprivation are not caused by contraction of the CAG repeat element in patients with systemic AID.

Patients with SLE and RA exhibit high levels of PPP2R2B CPG methylation. PPP2R2B contains a relatively large $\mathrm{CpG}$ island (i.e., CpG-rich area of the genome) that encompasses its first exon and 5' UTR as well as parts of the adjacent first intron (Figure 4A). The regulatory importance of this region is further suggested by the presence of 2 areas that exhibit DNAse I hypersensitivity in human T cells (16). Local CpG methylation has been associated with impaired B55 $\beta$ expression in human colorectal cancer (17). Therefore, we hypothesized that methylation of the PPP $2 R 2 B \mathrm{CpG}$ island could inhibit $\mathrm{B} 55 \beta$ transcription in $\mathrm{T}$ cells from patients with AID.

To test this hypothesis, we performed methylation-specific PCR, in which 2 sets of primers are used to interrogate the local methylation status in bisulfite-converted genomic DNA. One set of primers will amplify only when local cytosines are not methylated (U), and the other will amplify only when cytosines are methylated $(\mathrm{M})(17)$. The ratio of the products of each reaction is an indicator of local methylation. As shown in Figure 4B, DNA from $\mathrm{T}$ cells obtained from HDs amplified almost exclusively with primers specific for U DNA. In contrast, bands of variable intensity were detected when DNA from patients with SLE and RA was used, indicating the presence of local cytosine methylation (Figure 4B). Densitometric analysis revealed that DNA from patients with SLE and RA had significantly higher PPP2R $2 B$ CpG methylation than HDs (HDs $4.9 \pm 1.3$ vs. SLE $27.2 \pm 4.2 ; P=0.0002$; HD vs. RA $19.2 \pm 5.1 ; P=0.02$; Figure 4 C).

These results indicate that cytosine methylation is increased at the level of a conserved regulatory region of the PPP2R $2 B$ gene in patients with AID and suggest that transcription of B55 $\beta$ may be inhibited by this epigenetic mechanism in $\mathrm{T}$ cells in the setting of autoimmunity and/or systemic inflammation. 
A

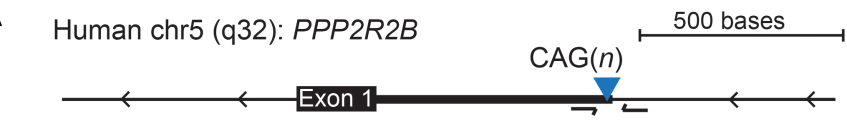

B
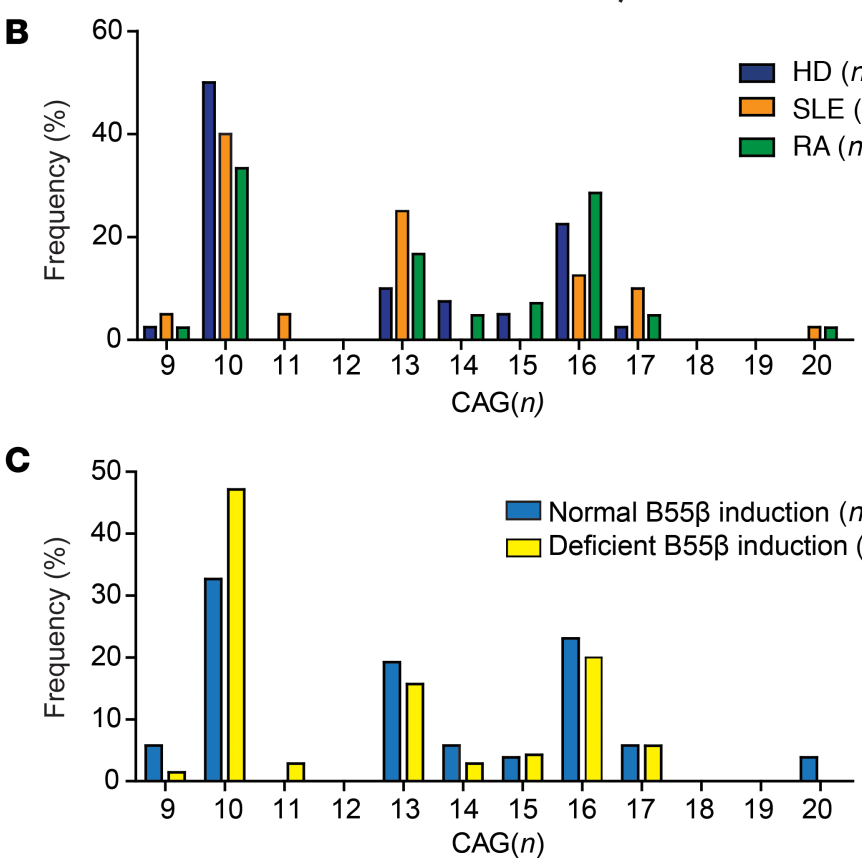

Figure 3. Defects in B55 $\beta$ upregulation are not caused by expansion or contraction of the CAG trinucleotide repeat in PPP2R2B. (A) Schematic representation of the genomic location of the first exon and 5 ' untranslated region (UTR) of PPP2R2B. The location of the CAG repeats and of the primers used to quantify the number of repeats in each individual are indicated by an inverted triangle and by arrows, respectively. (B) The distribution of allele length in HDs and patients with SLE and RA is shown (HD $n=20$; SLE $n=20$; RA $n=21$ ). (C) CAG repeat length in individuals who exhibited normal versus abolished upregulation of B $55 \beta$ during cytokine withdrawal was compared (normal B55 $\beta$ induction $n=21$; deficient $B 55 \beta$ induction $n=26$ ).

Methylation status of specific CpGs modulates B55 $\beta$ induction in response to cytokine withdrawal. To determine the contribution of individual cytosines to the regulation of B55 $\beta$ expression in patients with AID, we generated 5 sets of primers designed to quantify $\mathrm{CpG}$ methylation at the single base level using pyrosequencing (18) (Figure 5A). We observed that in HDs, the level of methylation throughout the CpG island is highly variable, ranging from $5 \%$ to $30 \%$. However, the methylation of individual cytosines tended to be consistent among different donors. We compared the methylation of each $\mathrm{CpG}$ between HDs and patients with RA and SLE. Methylation of the cytosines within the first amplicon (Amp), the one most proximal to the $P P P 2 R 2 B$ first exon, was significantly higher in patients with AID than in HDs (AID vs. HDs, $P=1.5$ $\times 10^{-5}$ ). The differences were also significant when patients with RA (RA vs. HDs, $P=6.5 \times 10^{-6}$ ) or SLE were analyzed separately (SLE vs. HD, $P=1.0 \times 10^{-4}$ ). No differences were observed in CpG methylation in Amps 2 to 5 between patients with AID and HDs (Figure 5, B and C).

Unsupervised hierarchical clustering, considering relative methylation of cytosines within Amps 1-3, segregated the samples into 3 clusters (Supplemental Figure 2). Except for 1, all HDs were included in clusters 1 and 2 . In contrast, more than half of patients were allocated to cluster $3(P<0.001$, Supplemental Figure 2$)$. This clustering, however, did not discriminate between donors with normal and abnormal induction of B55 $\beta$. Because most differences were observed at the level of Amp 1, we applied unsupervised hierarchical clustering to the samples according to methylation of Amp 1 (Figure 5D). Two clusters were distinguished: cluster 1 with low methylation and cluster 2 with higher methylation. Except for 1, all HDs were assigned to cluster $1(P<$ 0.001). In this case, the clustering also segregated samples according to B55 $\beta$ induction (B55 $\beta$ expression 24 hours after IL-2 withdrawal). All but 3 samples from cluster 1 exhibited normal B55 $\beta$ induction following IL-2 deprivation $(P<0.001)$ (Figure 5, D and $\mathrm{E})$.

Collectively, these data indicate that, in human $\mathrm{T}$ cells, local $\mathrm{CpG}$ methylation exerts a regulatory influence on $P P P 2 R 2 B$ transcription and indicate that under pathological autoimmunity, increased $\mathrm{CpG}$ methylation impedes B55 $\beta$ expression. 
$\mathbf{A}$

Human chr5 (q32): PPP2R2B

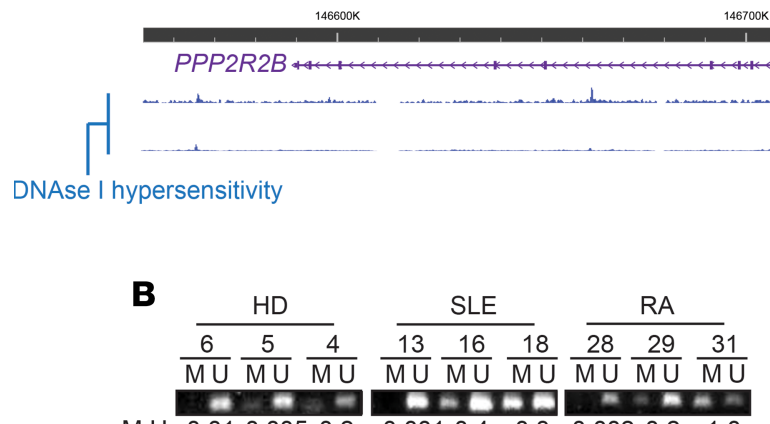

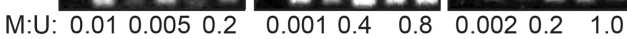
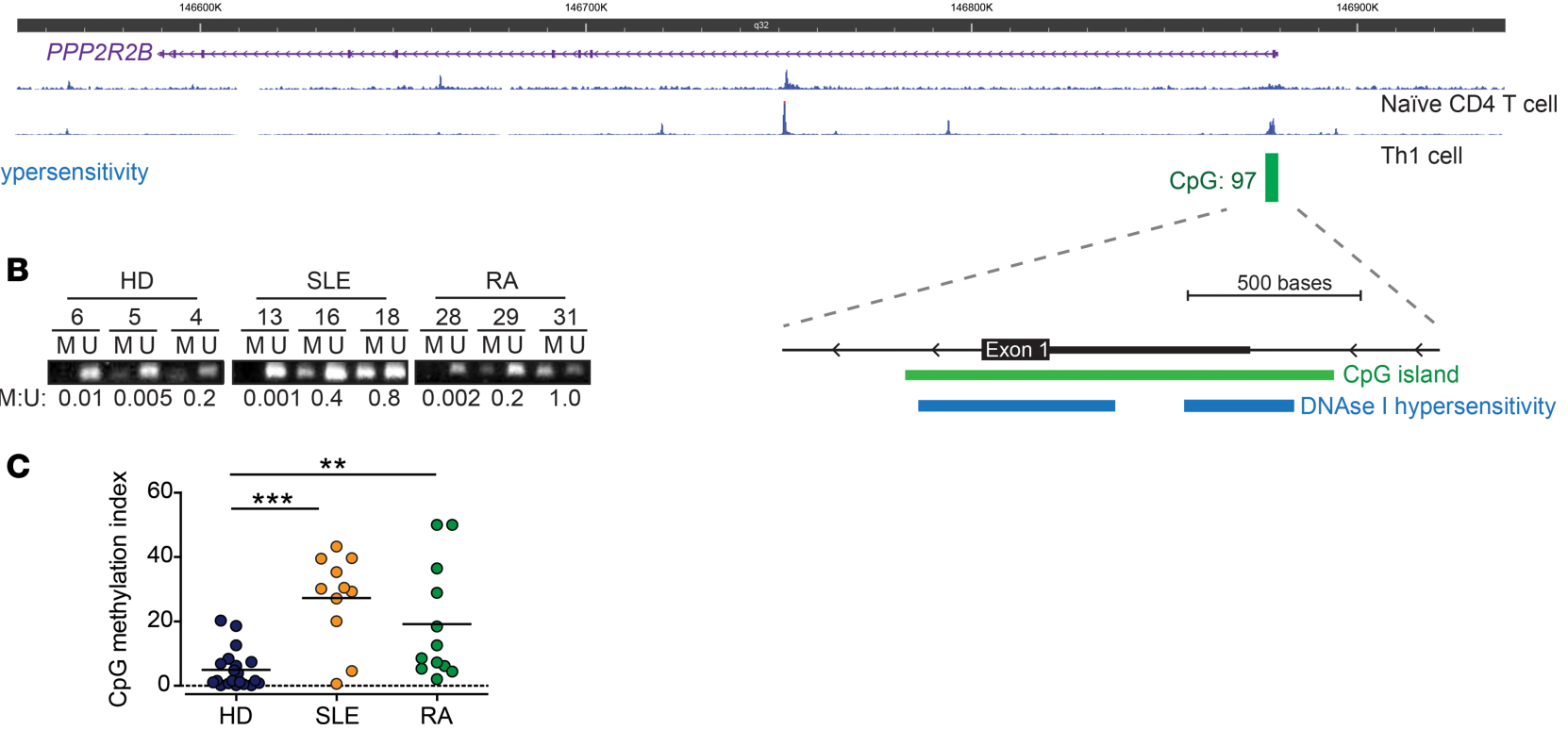

Figure 4. Local CpG DNA methylation is abnormally increased in patients with SLE and RA at the PPP2R2B locus. (A) Schematic representation of PPP2R2B, indicating the location of DNAse I hypersensitivity sites detected in human naive CD4+ $T$ cells and Th1-differentiated CD4+ $T$ cells (16). Also, the location of a large CpG island, composed of $97 \mathrm{CpG}$ dinucleotides, that encompasses the first exon and the $5^{\prime}$ UTR of the gene is shown. (B) Methylation-specific PCR was performed in T cell genomic DNA after bisulfite conversion. Shown are representative results of 3 HDs, 3 patients with SLE, and 3 patients with RA. M, methylated; U, unmethylated. Band density was quantified and the M/U ratio of each sample is shown. (C) Cumulative data from B presented as CpG methylation index: (M band $+\mathrm{U}$ band) $/ \mathrm{M}$ band. HD $n=20$; SLE $n=11 ; \mathrm{RA} n=12$. ${ }^{* *} P<0.01 ;{ }^{* * *} P<0.001$; 1-way ANOVA with Tukey's multiple-comparisons test.

Systemic inflammation is associated with PPP2R2B hypermethylation. Because defective B55 $\beta$ expression was not linked to a genetic variant, but rather to an epigenetic modification present in roughly $50 \%$ of patients with systemic autoimmunity (Figure $1 \mathrm{C}$ ), we hypothesized that inflammation may influence $P P P 2 R 2 B$ transcription by modifying the local epigenetic landscape. To assess the relationship between systemic inflammation and methylation of the $P P P 2 R 2 B C p G$ island, we compared the erythrocyte sedimentation rate (ESR) between patients with SLE and RA segregated to clusters 1 and 2 (Figure 5D). As shown in Figure 6A, ESR was significantly higher in patients from cluster 2 than in patients from cluster $1(32.1 \pm$ 8.0 vs. $6.7 \pm 1.4$, respectively; $P=0.01$ ). In fact, all patients from cluster 1 had normal levels of ESR, whereas $70 \%$ of patients from cluster 2 had high ESR $(P<0.001$, Fisher's exact test). Moreover, a significant correlation between ESR and $P P P 2 R 2 B$ methylation was observed (Figure $6 \mathrm{~B} ; r=0.758$, and $P=0.01$ ). In accordance with these findings, all patients with active RA (Disease Activity Score-28 [DAS28] > 2.6) were included in cluster 2 , while the only 2 patients assigned to cluster 1 were in clinical remission at the time of sample collection, with DAS28 scores lower than 2 (Figure 6C) (19). When we analyzed patients with SLE, we could not find any correlations between $P P P 2 R 2 B$ methylation and disease activity (assessed by SLE Disease Activity Index [SLEDAI]) (20) or target organ involvement (Figure 6D and data not shown).

$T N F-\alpha$ promotes $P P P 2 R 2 B$ methylation and impairs $C W I D$. The robust relationship between systemic inflammation and $P P P 2 R 2 B$ hypermethylation suggested that proinflammatory cytokines may mediate the epigenetic changes observed at the $P P P 2 R 2 B$ locus. To explore this possibility, we activated $\mathrm{T}$ cells from HDs in the presence of different cytokines (Figure 7A). We chose TNF- $\alpha$, IFN- $\alpha$, IL-6, IL-21, and IL-17 because they have been found to be abnormally increased in patients with RA or SLE $(21,22)$. Compared with T cells activated and expanded with only IL-2, T cells exposed to proinflammatory cytokines, except IL-17, acquired resistance to CWID (Figure 7B). However, qPCR revealed that B55 $\beta$ induction in response to cytokine withdrawal was not affected by IFN- $\alpha$, IL-6, and IL-17 and was augmented in cells exposed to IL-21. In sharp contrast, TNF- $\alpha$ significantly decreased basal levels of B55 $\beta$ and dampened its upregulation in response to cytokine deprivation (Figure 7C). These results indicate that inflammatory cytokines can promote resistance to apoptosis induced by cytokine withdrawal through different mechanisms and suggest that TNF- $\alpha$ may do so by repressing B55 $\beta$ expression. 
A

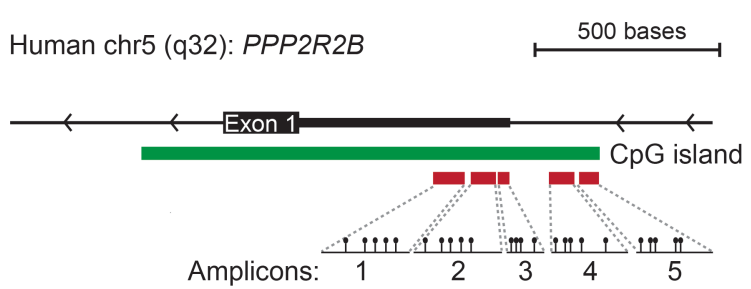

B

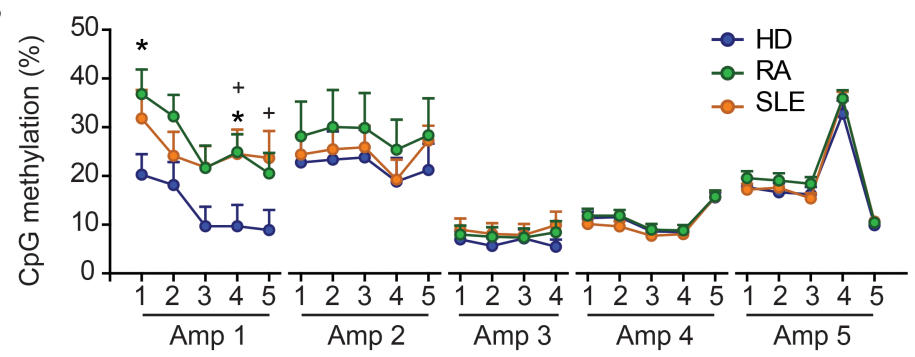

C

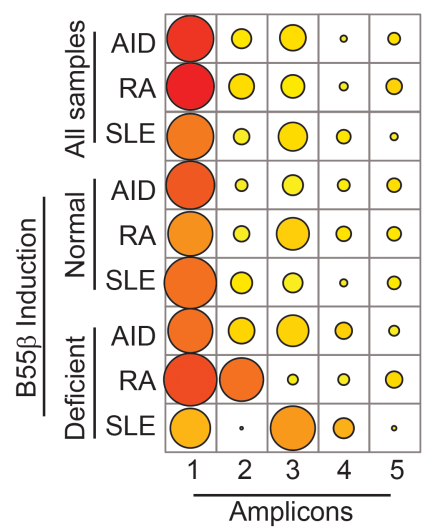

D

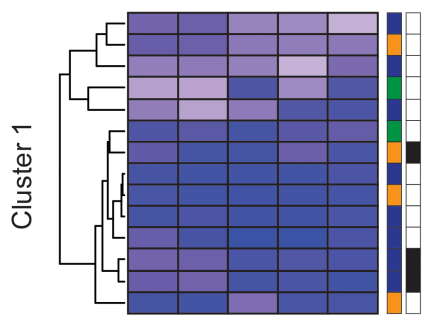

$\frac{N}{\frac{\Phi}{D}}$

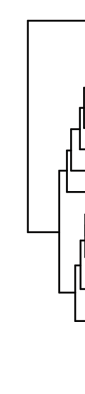

E
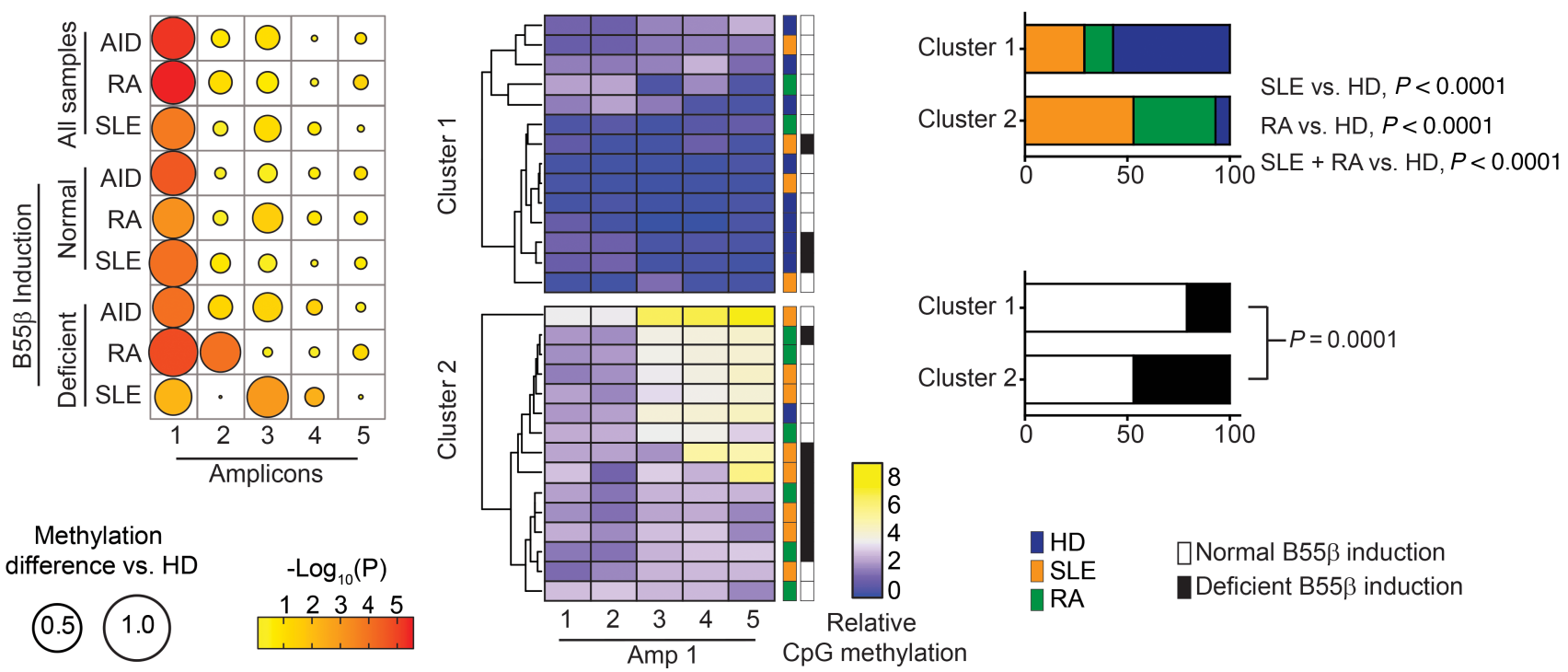

$\square$ Normal B55 $\beta$ induction

Deficient B55 $\beta$ induction

Figure 5. Methylation of discrete cytosines regulates B55 $\boldsymbol{\beta}$ expression in patients with SLE and RA. (A) Schematic representation of $P P P 2 R 2 B$, indicating the location of the $\mathrm{CpG}$ dinucleotides examined by pyrosequencing. (B) Methylation (percentage) of specific CpG dinucleotides in $\mathrm{T}$ cells isolated from HDs, patients with SLE, and patients with RA (HD $n=9-20 ;$ SLE $n=12-20 ;$ RA $n=8-20)$. RA vs. HD, ${ }^{*} P<0.05 ;$ SLE vs. HD, $+P<0.05 ; 2-$ way ANOVA with Tukey's multiple-comparisons test. (C) Differences in the DNA methylation between patients and controls. The diameters of each circle represent the difference between methylation in HDs and the indicated population. The color of each circle indicates the $P$ value of the corresponding comparison. Large differences were found in Amp 1. In addition, significant methylation differences were found in Amp 2 in patients with RA and in Amp 3 in patients with SLE. (D) Heatmap showing relative methylation (fold change over the mean of HDs) of the CpC dinucleotides from Amp 1. Samples were ordered by unsupervised clustering. The colored boxes on the right side of the heatmap indicate whether the sample corresponds to an HD or a patient; the black and white boxes indicate B55 $\beta$ induction after cytokine withdrawal. (E) Segregation of HDs and patients in clusters 1 and 2 (upper) and segregation of individuals with normal or defective B55 $\beta$ induction (lower). $P$ values were calculated using $\chi^{2}$ test.

Because TNF- $\alpha$ could inhibit B55 $\beta$ transcription by inducing local CpG methylation, we analyzed the methylation of the CpGs from Amp 1 in healthy T cells exposed to TNF- $\alpha$ and IL-21 and compared it with that of T cells expanded in IL-2. As shown in Figure 7, D and E, TNF- $\alpha$ significantly increased PPP2R2B methylation at the level of Amp 1. In contrast, IL-21 had no effect.

PPP2R2B hypermethylation inhibits CTCF binding to its target region. To clarify the mechanism by which $P P P 2 R 2 B$ methylation prevents B55 $\beta$ expression in $\mathrm{T}$ cells deprived of IL-2, we analyzed the sequence of Amp 1 using the Tomtom motif comparison tool (23) (Figure 8A). A putative binding site for CTCF was detected in Amp $1\left(P=2 \mathrm{e}^{-4}\right.$; Figure $\left.8 \mathrm{~B}\right)$. CTCF can act as a transcriptional activator or repressor or as an insulator, depending on the genomic context. Moreover, its binding is affected by local DNA methylation status (24). To determine whether CTFC binds to the $P P P 2 R 2 B$ promoter in activated $\mathrm{T}$ cells, we performed ChIP-qPCR assays. To this end, we compared local CTCF binding in healthy $\mathrm{T}$ cells activated and expanded in the absence or presence of TNF- $\alpha$. As shown in Figure $8 \mathrm{C}$, CTCF was found bound to the sequence of Amp 1 in activated T cells. However, exposure to TNF- $\alpha$ significantly reduced its binding.

Next, we compared the binding of CTCF to $P P P 2 R 2 B$ in $\mathrm{T}$ cells isolated from HDs and patients with AID. As shown in Figure 8D, abundance of CTCF at the $P P P 2 R 2 B$ promoter was significantly lower in T cells from patients with RA and SLE than in T cells from HDs. These results suggest that 
A

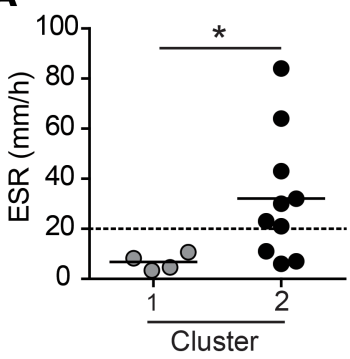

C

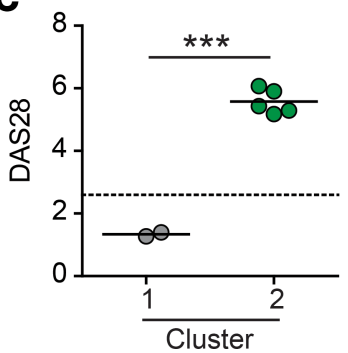

B

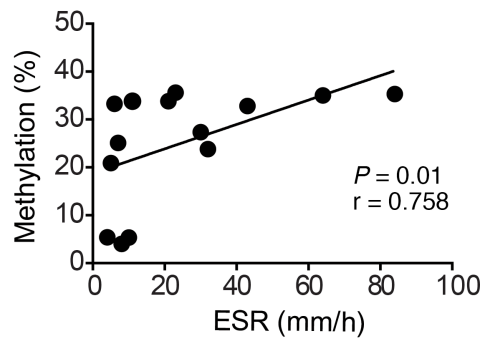

D

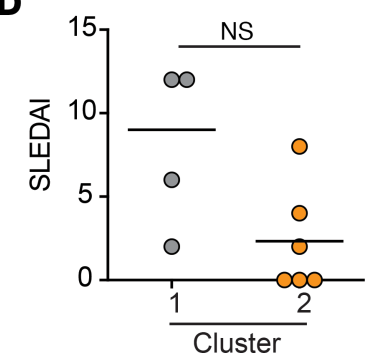

Figure 6. Methylation of PPP2R2B is associated with systemic inflammation. (A) Erythrocyte sedimentation rate (ESR), at the time of sample collection, in patients assigned to clusters 1 and $2 .{ }^{*} P=0.02$, Mann-Whitney $U$ test. (B) Correlation between ESR and CpG methylation (Amp 1) in patients with SLE and RA. Spearman's $r=$ 0.758 , and $P=0.01$. (C) Disease activity score in 28 joints (DAS28) in patients with RA assigned to clusters 1 and 2. ${ }^{* * *} P<0.0001$, unpaired two-tailed $t$ test. (D) SLE Disease Activity Index (SLEDAI) in patients with SLE from clusters 1 and 2 . NS, not significant (unpaired two-tailed $t$ test).

CTCF bound to the PPP2R $2 B$ promoter may facilitate $B 55 \beta$ expression and that the inflammatory milieu associated with systemic autoimmunity may interfere with its binding through methylation of the $P P P 2 R 2 B$ promoter region.

\section{Discussion}

Here, we show that B55 $\beta$ expression is altered in patients with systemic AID and that such defect is associated with resistance to CWID. Additionally, we provide evidence to demonstrate that the impaired upregulation of $B 55 \beta$ in response to cytokine deprivation is caused by hypermethylation of specific cytosines located within a regulatory element of $P P P 2 R 2 B$ that prevents the binding of CTCF. Finally, we identify systemic inflammation as a factor strongly associated with $P P P 2 R 2 B$ hypermethylation and demonstrate that a proinflammatory cytokine is able to induce $P P P 2 R 2 B$ epigenetic remodeling and resistance to CWID in healthy $\mathrm{T}$ cells. Collectively, our results reveal a gene whose expression is affected by an acquired defect through an epigenetic mechanism, in the setting of systemic autoimmunity. Because failure to remove activated $\mathrm{T}$ cells through CWID could contribute to autoimmune pathology, this mechanism represents a vicious cycle through which autoimmune inflammation contributes to its perpetuation (Figure 9).

Current paradigms assume that risk-conferring genetic variants contribute to the development of AID by promoting pathogenic responses when triggered by environmental factors (e.g., viruses) (10). PPP $2 R 2 B$ has not been associated with any AID, and therefore defects in its transcriptional regulation could not be attributed to pathological genetic variants in linkage disequilibrium with common single nucleotide polymorphisms. Because PPP2R2B contains a variable-length CAG repeat element that affects the expression of B55 $\beta$ (12), we sought associations between failed mRNA upregulation and differences in CAG repeat length. These were not found, suggesting that a fixed genetic element did not underlie the defective expression of B55 3 .

Methylation of cytosines located in $\mathrm{CpG}$ islands exerts suppressive effects on the transcription of neighboring genes (25). Because a substantial proportion of the causality of AID remains unexplained by risk loci identified in GWAS, efforts have been made to determine the role of epigenetic variation in autoimmune conditions (26). In fact, altered patterns of DNA methylation have been observed in patients with SLE $(27,28)$ and RA (29), suggesting that gene expression could be altered in patients with AID in an acquired manner. Inflammation is known to affect the phenotype and behavior of lymphocytes and other 
A<smiles>[3H]C(=C)CC1CCCCC(C=C)C1C(C)(C)C</smiles>

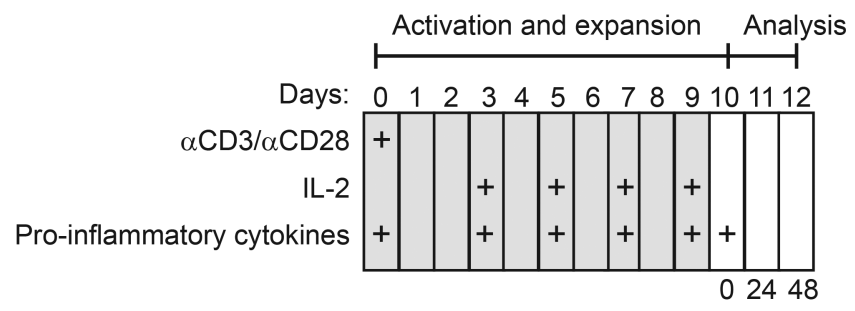

IL-6

IL-21

IL-17

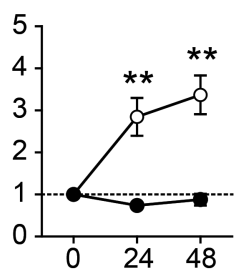

IL-2 withdrawal (h)

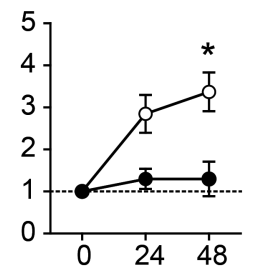

IL-21

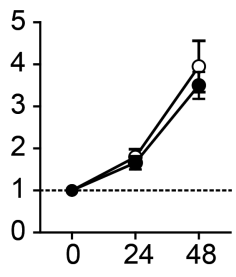

IL-17
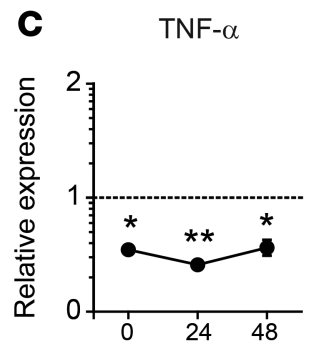

IFN- $\alpha$

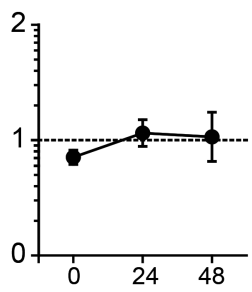

IL-6

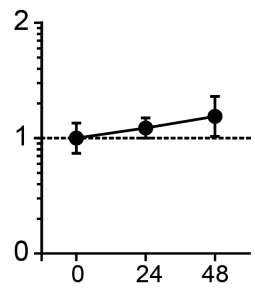

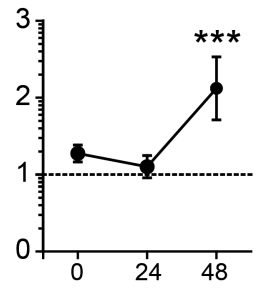

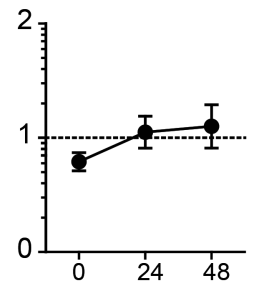

- No cytokine

- Cytokine

IL-2 withdrawal (h)

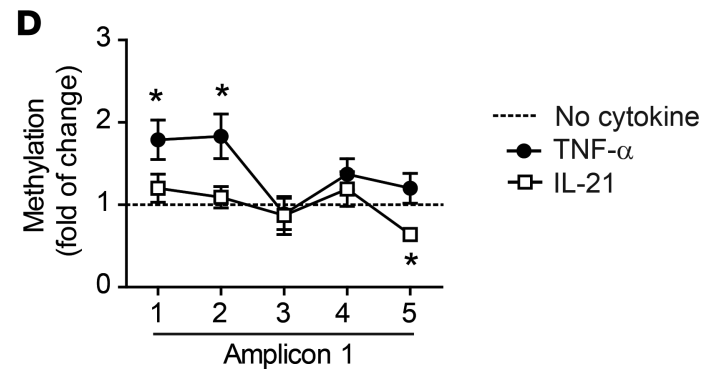

E

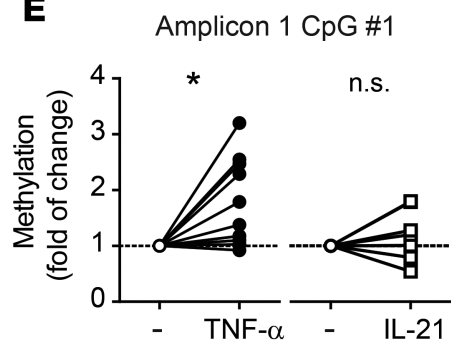

Amplicon $1 \mathrm{CpG} \# 2$

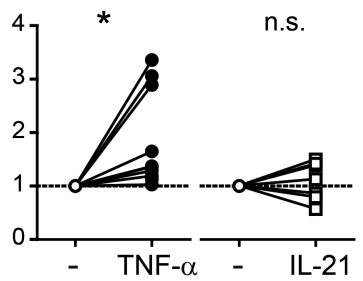

Figure 7. TNF- $\alpha$ induces PPP2R2B methylation, abolishes B55 $\beta$ expression, and impairs CWID in healthy T cells. (A) T cells from HDs were activated and expanded in the presence of IL-2 for 10 days. In addition to IL-2, at days $0,2,4,6$, and 8 , the indicated cytokines (TNF- $\alpha$, IFN- $\alpha$, IL-6, IL-21, or IL-17) were added to the culture. At day 10, cells were counted, washed, and replated in the absence of IL-2 and proinflammatory cytokines. (B) Apoptosis (annexin V+ SYTOX Orange-) was quantified before ( 0 hours) and after IL-2 withdrawal $(n=3-6)$. ${ }^{*} P<0.05 ;{ }^{*} P<0.01 ; 2$-way ANOVA with Tukey's multiple-comparisons test. (C) Expression of $B 55 \beta$ was determined ( $q$ PCR) before and after ( 24 and 48 hours) IL-2 withdrawal. Results were normalized against $A C T B$ (Ct) and then against cells expanded in the presence of IL-2 but in the absence of other cytokines $(\Delta \Delta \mathrm{Ct}){ }^{*} P<0.05 ;{ }^{* *} P<0.01 ;{ }^{* *} P<0.001 ; 2$-way ANOVA. (D) Methylation of the CpG dinucleotides from Amp 1 was determined by pyrosequencing in cells expanded in IL-2 (dotted line) and compared with the CpG DNA methylation of the same cells expanded in the presence of TNF- $\alpha$ or IL-21 $(n=8-11)$. ${ }^{*} P<0.05$, paired two-tailed $t$ test. (E) The relative change in methylation status of single CpC dinucleotides in response to TNF- $\alpha$ and IL-21 is shown $(n=8-11) .{ }^{*} P<0.05$, paired two-tailed $t$ test.

immune cells through epigenetic remodeling during acute immune responses induced by infectious agents $(30,31)$, but little is known about the effects imposed by chronic inflammation in the setting of AID. It is reasonable to assume that the abnormal phenotype exhibited by immune cells from patients with systemic AID could be caused by the superimposition of inflammation-induced epigenetic modifications on disease-associated genetic variants. Here, we have identified a gene that becomes silenced in patients with AID and have shown that the resulting phenotype — resistance to CWID — can be induced in healthy T cells by exposure to the proinflammatory cytokine TNF- $\alpha$.

We observed differences in the frequency of the B55 $\beta$ induction defect between the 3 systemic AID that we studied. We believe that these do not result from differential epigenetic regulation, but rather reflect 
A

Human chr5 (q32): PPP2R2B

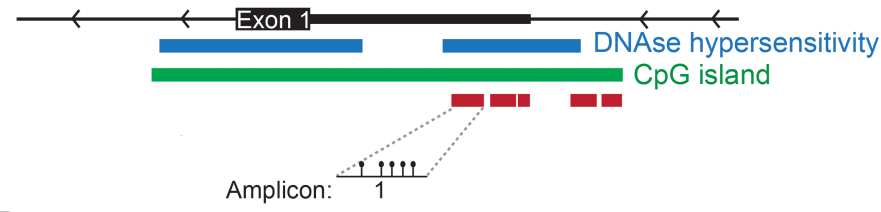

B

CTCF motif

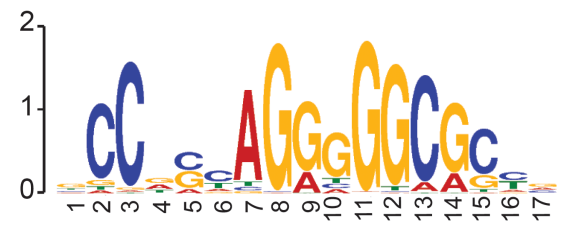

$p=2.0 \mathrm{e}^{-04}$

$E=8.06 \mathrm{e}^{-02}$

$q=7.81 \mathrm{e}^{-02}$

C

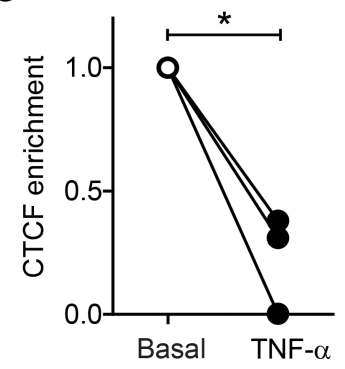

D

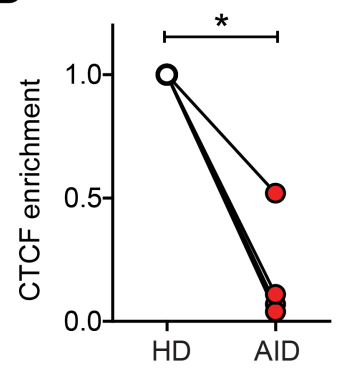

Figure 8. CTCF binding to PPP2R2B is decreased in T cells of patients with AID. (A) Schematic representation of $P P P 2 R 2 B$, indicating the location of the region that was analyzed (Amp 1). (B) CTCF motif present in Amp 1. (C) Quantification (ChIP-qPCR) of CTCF binding at Amp 1 in healthy T cells activated and expanded (basal) versus T cells expanded in the presence of TNF- $\alpha, n=3$. (D) CTCF binding to Amp 1 in activated T cells for HDs and patients with AID, $n=4$. Relative CTCF enrichment was normalized against basal in $\mathbf{C}$ or HDs in $\mathbf{D}$. Results are expressed as mean + SEM. ${ }^{*} P<0.05$, paired two-tailed $t$ test.

qualitative and quantitative differences in the systemic inflammation of patients with SLE, RA, and SS. This argument is based on the following: (a) there are no genetic associations between $P P P 2 R 2 B$ and these 3 AID; (b) careful examination of the $P P P 2 R 2 B C p G$ methylation status did not reveal disease-specific defects; (c) there is a robust association between systemic inflammation and $P P P 2 R 2 B$ methylation; (d) the epigenetic changes TNF- $\alpha$ induced in T cells from HDs were indistinguishable from the findings in $\mathrm{T}$ cells from patients with AID.

TNF- $\alpha$ has a well-established pathogenic role in RA, and its therapeutic blockade represents an effective therapy able to decrease disease activity (32) and induce clinical remission (33). In SLE, serum levels of TNF- $\alpha$ have been associated with disease activity (34), and TNF- $\alpha$ signaling has been proposed to contribute to inflammation at the level of specific tissues, in particular the kidney (35). Our results show that methylation at the $P P P 2 R 2 B$ locus is associated with presence of AID and with systemic inflammation, manifested by high ESR. In patients with RA, where disease activity is closely associated with systemic inflammation, we observed a robust correlation between DAS28 and PPP2R2B hypermethylation. These data are concordant with the capacity of TNF- $\alpha$ to induce epigenetic changes and repression of B55 $\beta$ transcription. On the other hand, SLE is a heterogeneous disease less strongly associated with inflammation. Although our sample size is limited, we did not observe any correlation between SLE disease activity and $P P P 2 R 2 B$ methylation. However, B55 $\beta$ expression and epigenetic landscape were clearly different between patients with SLE and HDs. This suggests that other autoimmunity-associated factors, aside from systemic inflammation, may modulate $P P P 2 R 2 B$ transcription. Of note, we found no correlation between the induction of $\mathrm{B} 55 \beta, \mathrm{CWID}$, or $P P P 2 R 2 B$ methylation patterns and demographic or clinical parameters, including age, sex, and medications; however, the number of patients included in this study precludes us from establishing definitive conclusions in this regard. 

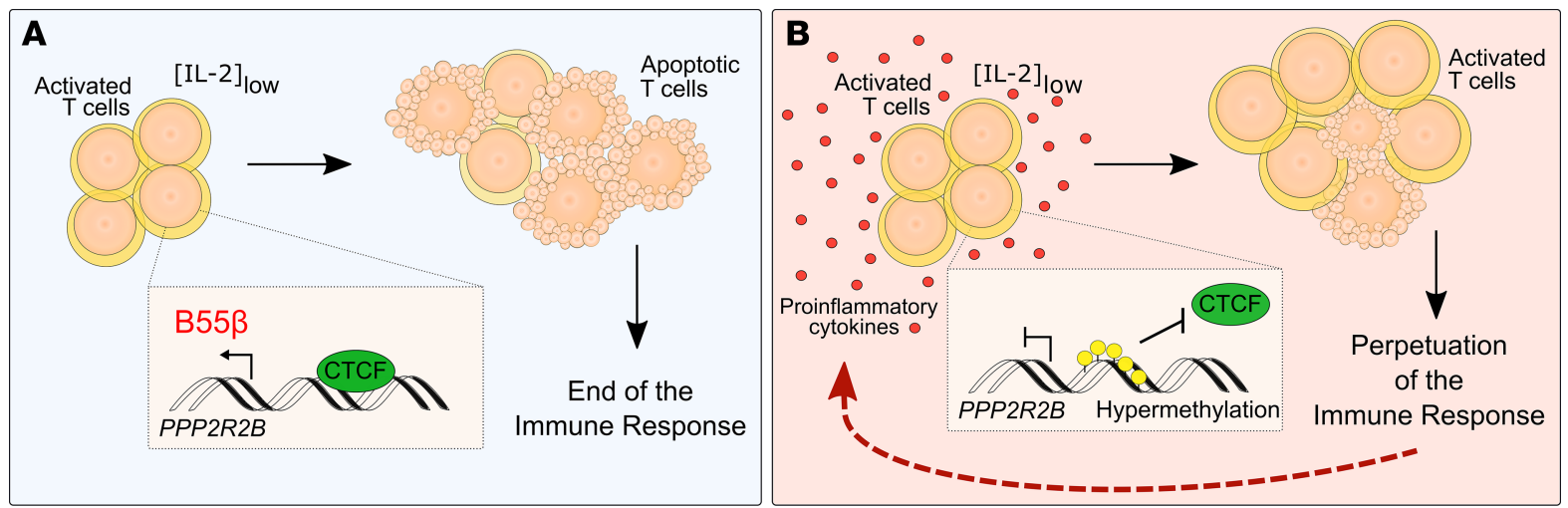

Figure 9. Mechanism through which PPP2R2B modulates the perpetuation of T cells in patients with AID. (A) After activation and expansion of healthy T cells, levels of IL-2 decrease, promoting clonal contraction by apoptosis. Low IL-2 concentration induces the expression of B55 $\beta$ through the binding of CTCF to a motif located within the CpG island of the PPP2R2B promoter. This mechanism promotes the termination of the immune response. (B) In the context of autoimmunity, where T cells are exposed to a proinflammatory environment, $P P P 2 R 2 B$ becomes hypermethylated, preventing the binding of CTCF. This impairs the induction of B55 $\beta$ and apoptosis of T cells when IL-2 levels are low. In consequence, survival of self-reactive activated T cells is facilitated and the autoimmune response is perpetuated.

CTCF is a ubiquitously expressed transcription factor that has been implicated in inflammatory processes. In CD4 ${ }^{+} \mathrm{T}$ cells, CTCF regulates the expression of IL-21 (36) and promotes the DNA hydroxymethylation of SOCS1 in cells of patients with SLE (37). In hippocampal neurons, lack of CTCF is associated with the dysregulation of a wide range of inflammation-related genes (e.g., Ccl2, Ccl3, Cd14, Ptgs2, Tlr2), suggesting that this transcription factor curbs neurological inflammation (38). In our study we demonstrated that CTCF binds to the PPP2R2B promoter, allowing the expression of B55 $\beta$ during IL-2 withdrawal. The autoimmune environment limits the binding of CTCF, probably through local DNA methylation, and therefore B55 $\beta$ transcription is impaired. These data support a methylation-sensitive association of CTCF with the $P P P 2 R 2 B$ promoter.

Apoptosis represents a fundamental process that controls $\mathrm{T}$ cell numbers and activity. Throughout their lifespan, T cells rely on the presence of different stimuli to inhibit apoptosis and survive (39). Resting cells (naive and memory) require low-affinity TCR signaling and the presence of prosurvival cytokines (e.g., IL-7). In contrast, activated T cells are highly susceptible to apoptosis induced through Fas and triggered by cytokine withdrawal (CWID) (40). Here, we show that CWID in activated T cell blasts from HDs and patients with AID is highly dependent on B55 $\beta$. Freshly isolated human CD4+ $\mathrm{T}$ cell subsets (i.e., naive, effector memory, and central memory) express relatively low levels of B55 $\beta$. In contrast, B55 $\beta$ expression in previously activated $\mathrm{CD} 8^{+} \mathrm{T}$ cells is higher than in naive $\mathrm{CD} 8^{+} \mathrm{T}$ cells (Supplemental Figure 3 ). TCR-mediated activation induces the downregulation of B55 $\beta$. This may facilitate cell cycle entry or promote the metabolic changes associated with blast transformation. The importance of B55 $\beta$ in regulating $\mathrm{T}$ cell quiescence and its role in defining susceptibility to CWID in different $\mathrm{T}$ cell subsets is the focus of active investigation.

The relationship between PP2A and apoptosis has been known for a long time $(6,41)$. PP2A can activate a number of proapoptotic molecules, including FoxO1 (42) and members of the Bcl-2 family (41). However, the mechanisms through which induction of B55 $\beta$ triggers apoptosis is not understood, and, in general, little is known about how specific PP2A holoenzymes affect apoptosis and how different PP2A regulatory subunits modulate this process. B55 $\beta$ is particularly interesting because it is modulated at the transcriptional level. In contrast, most PP2A B subunits are constitutively expressed and regulated at the posttranslational level (7). B55 $\beta$ is downregulated by TCR signaling and its de novo synthesis, is induced by cytokine withdrawal, and is intimately associated with apoptosis (7).

Apoptosis of activated $\mathrm{T}$ cells represents an essential process through which the immune system eliminates expanded clones of effector cells that are no longer needed. This allows the immune system to return to its basal state and to rebalance its $\mathrm{T}$ cell receptor repertoire (43). The correct termination of immune responses diminishes the risk of immunopathology and autoimmunity that activated $\mathrm{T}$ cells entail. CWID is considered the main trigger of $\mathrm{T}$ cell death during the contraction phase that curtails clonal expansion, and, accordingly, genetic defects in molecules involved in CWID have been associated with autoimmunity (44). Defects in T cell apoptosis have been documented in patients with SLE $(7,45,46)$ and RA (47) 
through different mechanisms. Our data illustrate a mechanism through which apoptosis resistance can be acquired by activated $\mathrm{T}$ cells through the epigenetic remodeling of the $P P P 2 R 2 B$ locus. This phenomenon could contribute to the increased abundance of activated T cells observed in SLE and RA $(48,49)$.

In summary, our work identifies a mechanism through which chronic inflammation affects the expression of a gene that plays a central role during the termination of the immune response. This illustrates how a pathological environment can affect in a noxious manner the behavior of $\mathrm{T}$ lymphocytes, amplifying inflammation and contributing to the continuation of pathological autoimmunity.

\section{Methods}

Patients and HDs. Peripheral blood samples were obtained from HDs $(n=28)$ and patients with SLE $(n=$ $34)$, RA $(n=29)$, and SS $(n=25)$. The study was approved by the IRB of the Instituto Nacional de Ciencias Médicas y Nutrición Salvador Zubirán (IRE-1805). All participants signed informed consent forms. Patients with SLE fulfilled the 1997 American College of Rheumatology (ACR) classification criteria (50), patients with RA fulfilled the 2010 ACR/European League Against Rheumatism (ACR/EULAR) classification criteria (51), and patients with SS fulfilled the 2016 ACR/EULAR classification criteria (52). Age- and sex-matched HDs $(n=25)$ with no family history of AID were recruited as controls. The clinical and demographic characteristics of the study participants are summarized in Supplemental Table 1.

Isolation and expansion of T cells. Peripheral blood mononuclear cells (PBMCs) were obtained by Ficoll gradient centrifugation. T cells were separated from PBMCs by negative selection using MagniSort Human $\mathrm{T}$ cell enrichment kit (Thermo Fisher Scientific). Purity was higher than $96 \%$ in all cases. One million $\mathrm{T}$ cells were activated in the presence of plate-bound anti-CD3 (BioXCell, OKT3, $5 \mu \mathrm{g} / \mathrm{ml}$ ) and anti-CD28 (BioXCell, clone 9.3, $2.5 \mu \mathrm{g} / \mathrm{ml}$ ), in $0.5 \mathrm{ml}$ of RPMI (MilliporeSigma) supplemented with 10\% FCS (Thermo Fisher Scientific) and penicillin/streptomycin, at $37^{\circ} \mathrm{C}$ in an atmosphere of $5 \% \mathrm{CO}_{2}$. Three days later, cells were transferred into a new plate, culture medium was doubled, and IL-2 (Peprotech, $100 \mathrm{U} / \mathrm{ml}$ ) was added. Every 48 hours, supplemented RPMI and IL-2 were added, until day 10 (Supplemental Figure 1). For $\mathrm{T}$ cell activation and expansion in the presence of proinflammatory cytokines, one of the following was included from day 0: IFN- $\alpha$ (BioLegend, $1000 \mathrm{U} / \mathrm{ml}$ ), IL-6 (Peprotech, 25 ng/ml), IL-21 (Peprotech, 25 ng/ml), TNF- $\alpha$ (Peprotech, 20 ng/ml), or IL-17A (Peprotech, 5 ng/ml).

Apoptosis detection. At day 10 of activation, cells were washed and replated in fresh supplemented RPMI, free of IL-2 $\left(0.5 \times 10^{6}\right.$ cells $\left./ \mathrm{ml}\right)$. Apoptosis was assessed immediately (basal) and after 24 and 48 hours of IL-2 deprivation. Apoptosis was defined as annexin V (BioLegend) binding in the absence of membrane permeability to DAPI or SYTOX Orange (Thermo Fisher Scientific). Apoptotic CD4 ${ }^{+}$and $\mathrm{CD} 8^{+} \mathrm{T}$ cells were quantified by flow cytometry (anti-CD4, clone RPA-T4; anti-CD8, clone RPA-T8, both from Tonbo). Data were acquired on an LSR Fortessa cytometer (BD Biosciences) and analyzed using FlowJo (Tree Star, Inc.).

B55 $\beta$ expression. Total RNA was extracted from T cell blasts before (basal) and after ( 24 and 48 hours) IL-2 withdrawal using TRIzol (Thermo Fisher Scientific). cDNA was generated from $1 \mu \mathrm{g}$ of RNA using High-Capacity cDNA Reverse Transcription Kit (Thermo Fisher Scientific). SYBR Green-based qPCR was performed in triplicate using a QuantStudio 7 Flex Real-Time PCR System (Applied Biosystems). Ct values were normalized against $A C T B$. Results are expressed as $\triangle \triangle C$ t. Primer sequences are provided in Supplemental Table 3.

DNA extraction and bisulfite conversion. To obtain DNA, $0.8 \mathrm{ml}$ of lysis buffer (protein kinase $1 \times$ buffer $[0.075$ $\mathrm{M} \mathrm{NaCl}$ and $0.024 \mathrm{M}$ EDTA, $\mathrm{pH}=8], 10 \mathrm{mg} / \mathrm{ml}$ proteinase $\mathrm{K}$, and $5 \% \mathrm{SDS}$ ) was added to each sample and incubated at $55^{\circ} \mathrm{C}$ overnight. Samples were centrifuged and supernatants were transferred to a new tube with $0.2 \mathrm{ml}$ of $6 \mathrm{M} \mathrm{NaCl}$ added, vortexed, and centrifuged at 12,000 $\mathrm{g}$ for 10 minutes. The supernatant was recovered and $0.5 \mathrm{ml}$ of absolute ethanol was added and mixed by inversion. One milliliter of cold $70 \%$ ethanol was added to the DNA pellet, which was centrifuged at $12,000 \mathrm{~g}$ and allowed to dry. The DNA pellet was resuspended in $0.1 \mathrm{ml}$ of DNAse-free water and stored at $-80^{\circ} \mathrm{C}$ until use. For DNA bisulfite conversion, $1 \mu \mathrm{g}$ of genomic DNA was processed using EpiTect Bisulfite Kit (Qiagen), following the instructions of the supplier.

Quantification of $C A G$ repeat frequency. Analyses of the $P P P 2 R 2 B$ repeat length were performed by fragment length analysis. PCR was performed using previously reported primers (13) provided in Supplemental Table 3. Each reaction was performed with $0.30 \mathrm{ng}$ of DNA using HotStarTaq Master Mix following the instructions of the supplier (Qiagen) and an optimized $58^{\circ} \mathrm{C}$ touchdown protocol. One microliter of the amplified fragment, $17.6 \mu \mathrm{l}$ of HiDi formamide (Applied Biosystems, Thermo Fisher Scientific), and $0.4 \mu \mathrm{l}$ of GeneScan-500LIZ (Applied Biosystems, Thermo Fisher Scientific) standard size were mixed, denatured for 5 minutes, and immediately chilled on ice for a few minutes before fragment analysis. Analysis was performed on an AB 3500 Genetic 
Analyzer (Thermo Fisher Scientific) using the POP7 polymer. The fluorescent fragments were analyzed automatically using the SizeColler database v.1.1.0 and manually with GeneMapper v.5.1 (Thermo Fisher Scientific).

Methylation-specific PCR. Previously described primers (17), specific for the CpG island of the PPP2R2B promoter, were used to amplify bisulfite-modified DNA (Supplemental Table 3). PCR was performed on 30 ng of converted DNA, using HotStarTaq Master Mix (Qiagen). The PCR product was run on a $\%$ agarose gel, stained with ethidium bromide, and visualized in a transilluminator (Bio-Rad). The relative quantification of bands and $\mathrm{U}$ and $\mathrm{M}$ alleles was done using ImageJ64 (NIH).

Pyrosequencing. Five sets of primers, specific for the $\mathrm{CpG}$ island promoter sequence of $P P P 2 R 2 B$, were designed using the PyroMark Assay Design 2.0 program (Qiagen). A PCR reaction (20 $\mu 1$ total) was performed using PyroMark PCR Kit (Qiagen) on $2 \mu 1$ of bisulfite-converted DNA. Three microliters of each amplicon was analyzed by electrophoresis on a $2 \%$ agarose gel. The remaining product was mixed with binding buffer and sepharose beads (GE Healthcare) with streptavidin, incubated 15 minutes under constant agitation, washed with 70\% ethanol, and denatured with PyroMark denaturing solution. Hybridization was performed with the dilution sequencing probe in PyroMark alignment buffer for 2 minutes at $80^{\circ} \mathrm{C}$. The test was performed on a PyroMark Q24 pyrosequencer and analyzed using the Q24 2.0 program (Qiagen). The primer sequences (amplification and sequencing) are provided in Supplemental Table 3.

ChIP. T cells $\left(2.5 \times 10^{6}\right)$ were fixed with $1 \%$ formaldehyde and neutralized by adding $0.125 \mathrm{M}$ glycine. The cells were then lysed (25 mM Tris, $\mathrm{pH} 7.5 ; 150 \mathrm{mM} \mathrm{NaCl}, 5 \mathrm{mM}$ EDTA, 0.1\% Triton X-100, 1\% SDS, $0.5 \%$ deoxycholate, plus protease inhibitor cocktail [Roche]) and sonicated to obtain soluble chromatin with a length of 200-400 bp. ChIP was performed using an anti-CTCF antibody (07-729, MilliporeSigma). Immunoprecipitated chromatin was incubated with proteinase $\mathrm{K}(2 \mathrm{mg} / \mathrm{ml})$ and RNAse A (5 $\mathrm{mg} / \mathrm{ml})$ for 2 hours at $37^{\circ} \mathrm{C}$ followed by $65^{\circ} \mathrm{C}$ overnight. Then, chromatin was cleaned and concentrated using ChIP DNA Clean \& Concentrator kit (D5205, Zymo Research). Eluted DNA was subjected to reverse transcription PCR (RT-PCR) amplification. The primer sequences are provided in Supplemental Table 3.

Statistics. Statistical analyses were performed using GraphPad Prism 6.0 (GraphPad Software). Data are represented as mean \pm SEM. Mann-Whitney $U$ test or Student's $t$ test ( 2 tailed) were used for comparisons between groups. One-way ANOVA was used to determine statistical differences among 3 groups and 2-way ANOVA between groups with more than 2 variables; statistical test correction was applied as indicated in the figure legends. $P$ values less than 0.05 were considered significant.

Study approval. This study was approved by the IRB of the Instituto Nacional de Ciencias Médicas y Nutrición Salvador Zubirán (INCMNSZ; IRE-1805). All participants signed informed consent forms.

\section{Author contributions}

IKMS, NRR, OMM, JAV, FR, and JCC conceived the study; IKMS, BESH, YS, NRR, OMM, FR, and JCC developed the methodology; IKMS, BESH, YS, MEV, MITZ, HBGG, and GHM performed the experiments; BESH, JAV, OMM, FR, and JCC provided the resources. IKMS and JCC wrote the original draft; IKMS, OMM, JAV, FR, and JCC reviewed and edited the manuscript; BESH, JAV, and JCC acquired funding; FR and JCC supervised the study.

\section{Acknowledgments}

We thank the patients and the clinicians from the Department of Immunology and Rheumatology (INCMNSZ) for their participation in this study. We thank Araceli Martínez and Martha E. Baños (INCMNSZ) for their help procuring blood samples, Melitón Zúñiga (INCMNSZ) for his invaluable help in maintaining a clean and organized lab, Armando Gamboa from the Department of Pathology (INCMNSZ) for his help with pyrosequencing, José Jiram Torres-Ruiz (INCMNSZ) for his help in evaluating clinical scores, and Victor M. Ortiz-Ortega (INCMNSZ) for his help with DNA sonication. This work was supported by grants from the Consejo Nacional de Ciencia y Tecnología, Mexico (IFC 2015-549; FOSISS 2014-233667; FOSSIS 2016-272118; and CB 2015-256752).

Address correspondence to: José C. Crispín or Florencia Rosetti, Department of Immunology and Rheumatology, Instituto Nacional de Ciencias Médicas y Nutrición Salvador Zubirán, Vasco de Quiroga \#15, Mexico City, Mexico 14080. Phone: 5255.5487.0900 ext. 2610; Email: carlos.crispina@incmnsz.mx (JCC); florencia.rosettis@incmnsz.mx (FR). 
1. Flores-Mendoza G, Sansón SP, Rodríguez-Castro S, Crispín JC, Rosetti F. Mechanisms of tissue injury in lupus nephritis. Trends Mol Med. 2018;24(4):364-378.

2. Dominguez-Villar M, Hafler DA. Regulatory T cells in autoimmune disease. Nat Immunol. 2018;19(7):665-673.

3. McKinney EF, et al. A CD8+ T cell transcription signature predicts prognosis in autoimmune disease. Nat Med. 2010;16(5):586-91.

4. McKinney EF, Lee JC, Jayne DR, Lyons PA, Smith KG. T-cell exhaustion, co-stimulation and clinical outcome in autoimmunity and infection. Nature. 2015;523(7562):612-616.

5. Shi Y. Serine/threonine phosphatases: mechanism through structure. Cell. 2009;139(3):468-484.

6. Janssens V, Longin S, Goris J. PP2A holoenzyme assembly: in cauda venenum (the sting is in the tail). Trends Biochem Sci. 2008;33(3):113-121.

7. Crispín JC, Apostolidis SA, Finnell MI, Tsokos GC. Induction of PP2A B $\beta$, a regulator of IL-2 deprivation-induced T-cell apoptosis, is deficient in systemic lupus erythematosus. Proc Natl Acad Sci USA. 2011;108(30):12443-12448.

8. Crispín JC, Kyttaris VC, Juang YT, Tsokos GC. How signaling and gene transcription aberrations dictate the systemic lupus erythematosus T cell phenotype. Trends Immunol. 2008;29(3):110-115.

9. Delgado-Vega A, Sánchez E, Löfgren S, Castillejo-López C, Alarcón-Riquelme ME. Recent findings on genetics of systemic autoimmune diseases. Curr Opin Immunol. 2010;22(6):698-705.

10. Crispín JC, Hedrich CM, Tsokos GC. Gene-function studies in systemic lupus erythematosus. Nat Rev Rheumatol. 2013;9(8):476-484.

11. Snow AL, et al. Restimulation-induced apoptosis of T cells is impaired in patients with X-linked lymphoproliferative disease caused by SAP deficiency. J Clin Invest. 2009;119(10):2976-2989.

12. Lin $\mathrm{CH}$, et al. The CAG repeat in SCA12 functions as a cis element to up-regulate PPP2R2B expression. Hum Genet. 2010;128(2):205-212.

13. Holmes SE, et al. Expansion of a novel CAG trinucleotide repeat in the 5' region of PPP2R2B is associated with SCA12. Nat Genet. 1999;23(4):391-392.

14. Cohen RL, Margolis RL. Spinocerebellar ataxia type 12: clues to pathogenesis. Curr Opin Neurol. 2016;29(6):735-742.

15. Chen CM, et al. PPP2R2B CAG repeat length in the Han Chinese in Taiwan: association analyses in neurological and psychiatric disorders and potential functional implications. Am J Med Genet B Neuropsychiatr Genet. 2009;150B(1):124-129.

16. Thurman RE, et al. The accessible chromatin landscape of the human genome. Nature. 2012;489(7414):75-82.

17. Tan J, et al. B55 $\beta$-associated PP2A complex controls PDK1-directed myc signaling and modulates rapamycin sensitivity in colorectal cancer. Cancer Cell. 2010;18(5):459-471.

18. Harrington CT, Lin EI, Olson MT, Eshleman JR. Fundamentals of pyrosequencing. Arch Pathol Lab Med. 2013;137(9):1296-1303.

19. Fransen J, Stucki G, van Riel PLCM. Rheumatoid arthritis measures: Disease Activity Score (DAS), Disease Activity Score-28 (DAS28), Rapid Assessment of Disease Activity in Rheumatology (RADAR), and Rheumatoid Arthritis Disease Activity Index (RADAI). Arthritis Rheum. 2003;49(S5):S214-S224.

20. Gladman DD, Ibañez D, Urowitz MB. Systemic lupus erythematosus disease activity index 2000. J Rheumatol. 2002;29(2):288-291.

21. McInnes IB, Schett G. Cytokines in the pathogenesis of rheumatoid arthritis. Nat Rev Immunol. 2007;7(6):429-442.

22. Rönnblom L, Elkon KB. Cytokines as therapeutic targets in SLE. Nat Rev Rheumatol. 2010;6(6):339-347.

23. Gupta S, Stamatoyannopoulos JA, Bailey TL, Noble WS. Quantifying similarity between motifs. Genome Biol. 2007;8(2):R24.

24. Fang R, Wang C, Skogerbo G, Zhang Z. Functional diversity of CTCFs is encoded in their binding motifs. BMC Genomics. 2015;16:649.

25. Schübeler D. Function and information content of DNA methylation. Nature. 2015;517(7534):321-326.

26. Rakyan VK, Down TA, Balding DJ, Beck S. Epigenome-wide association studies for common human diseases. Nat Rev Genet. 2011;12(8):529-541.

27. Jeffries MA, Dozmorov M, Tang Y, Merrill JT, Wren JD, Sawalha AH. Genome-wide DNA methylation patterns in CD4+ T cells from patients with systemic lupus erythematosus. Epigenetics. 2011;6(5):593-601.

28. Zhu H, et al. Whole-genome transcription and DNA methylation analysis of peripheral blood mononuclear cells identified aberrant gene regulation pathways in systemic lupus erythematosus. Arthritis Res Ther. 2016;18:162.

29. Liu Y, et al. Epigenome-wide association data implicate DNA methylation as an intermediary of genetic risk in rheumatoid arthritis. Nat Biotechnol. 2013;31(2):142-147.

30. Henning AN, Roychoudhuri R, Restifo NP. Epigenetic control of CD8+ T cell differentiation. Nat Rev Immunol. 2018;18(5):340-356.

31. Lau CM, et al. Epigenetic control of innate and adaptive immune memory. Nat Immunol. 2018;19(9):963-972

32. Kavanaugh A, et al. Clinical, functional and radiographic consequences of achieving stable low disease activity and remission with adalimumab plus methotrexate or methotrexate alone in early rheumatoid arthritis: 26-week results from the randomised, controlled OPTIMA study. Ann Rheum Dis. 2013;72(1):64-71.

33. Breedveld FC, et al. The PREMIER study: a multicenter, randomized, double-blind clinical trial of combination therapy with adalimumab plus methotrexate versus methotrexate alone or adalimumab alone in patients with early, aggressive rheumatoid arthritis who had not had previous methotrexate treatment. Arthritis Rheum. 2006;54(1):26-37.

34. Idborg H, et al. TNF- $\alpha$ and plasma albumin as biomarkers of disease activity in systemic lupus erythematosus. Lupus Sci Med. 2018;5(1):e000260.

35. Qing X, et al. iRhom2 promotes lupus nephritis through TNF- $\alpha$ and EGFR signaling. J Clin Invest. 2018;128(4):1397-1412.

36. Park JH, Choi Y, Song MJ, Park K, Lee JJ, Kim HP. Dynamic long-range chromatin interaction controls expression of IL-21 in CD4+ T cells. J Immunol. 2016;196(10):4378-4389.

37. Zhao M, et al. Increased 5-hydroxymethylcytosine in CD4(+) T cells in systemic lupus erythematosus. J Autoimmun 2016;69:64-73.

38. McGill BE, et al. Abnormal microglia and enhanced inflammation-related gene transcription in mice with conditional deletion of Ctcf in Camk2a-Cre-expressing neurons. J Neurosci. 2018;38(1):200-219.

39. Nagata S, Tanaka M. Programmed cell death and the immune system. Nat Rev Immunol. 2017;17(5):333-340.

40. Zhan Y, Carrington EM, Zhang Y, Heinzel S, Lew AM. Life and death of activated T cells: how are they different from naïve T cells? Front Immunol. 2017;8:1809. 
41. Van Hoof C, Goris J. Phosphatases in apoptosis: to be or not to be, PP2A is in the heart of the question. Biochim Biophys Acta. 2003;1640(2-3):97-104.

42. Yan L, Lavin VA, Moser LR, Cui Q, Kanies C, Yang E. PP2A regulates the pro-apoptotic activity of FOXO1. J Biol Chem. 2008;283(12):7411-7420.

43. Marrack P, Scott-Browne J, MacLeod MK. Terminating the immune response. Immunol Rev. 2010;236:5-10.

44. Snow AL, Pandiyan P, Zheng L, Krummey SM, Lenardo MJ. The power and the promise of restimulation-induced cell death in human immune diseases. Immunol Rev. 2010;236:68-82.

45. Gergely P, et al. Mitochondrial hyperpolarization and ATP depletion in patients with systemic lupus erythematosus. Arthritis Rheum. 2002;46(1):175-190.

46. Xu L, Zhang L, Yi Y, Kang HK, Datta SK. Human lupus T cells resist inactivation and escape death by upregulating COX-2. Nat Med. 2004;10(4):411-415.

47. Schirmer M, Vallejo AN, Weyand CM, Goronzy JJ. Resistance to apoptosis and elevated expression of Bcl-2 in clonally expanded CD4+CD28- T cells from rheumatoid arthritis patients. J Immunol. 1998;161(2):1018-1025.

48. Crispin JC, Martínez A, de Pablo P, Velasquillo C, Alcocer-Varela J. Participation of the CD69 antigen in the T-cell activation process of patients with systemic lupus erythematosus. Scand J Immunol. 1998;48(2):196-200.

49. Ponchel F, et al. Dysregulated lymphocyte proliferation and differentiation in patients with rheumatoid arthritis. Blood. 2002;100(13):4550-4556.

50. Hochberg MC. Updating the American College of Rheumatology revised criteria for the classification of systemic lupus erythematosus. Arthritis Rheum. 1997;40(9):1725.

51. Aletaha D, et al. 2010 Rheumatoid arthritis classification criteria: an American College of Rheumatology/European League Against Rheumatism collaborative initiative. Arthritis Rheum. 2010;62(9):2569-2581.

52. Shiboski CH, et al. League Against Rheumatism classification criteria for primary Sjögren's syndrome: a consensus and data-driven methodology involving three international patient cohorts. Arthritis Rheumatol. 2017;69(1):35-45. 